\title{
Use Case Analysis for Standard Manufacturing Service Capability Model
}

\author{
Yunsu Lee, Boonserm (Serm) Kulvatunyou, Marko Vujasinovic, and Nenad Ivezic \\ Systems Integration Division, NIST, Gaithersburg, USA \\ \{yun-su.lee, serm, marko.vujasinovic, nivezic\} @nist.gov
}

\begin{abstract}
Manufacturing enterprises are becoming globally distributed production systems. Rigid supply chains are giving way to dynamic supply networks that are cost-efficient and can respond to change quickly. A key factor in the formation of dynamic supply networks is the communication of manufacturing capabilities - both production capabilities and information processing capabilities. These are collectively referred to as manufacturing service capability (MSC) information. Presently, MSC information is provided using many different, proprietary terminologies and representations. The lack of a standard model impedes communications of MSC information. We propose the development of a standard MSC model to enhance the MSC information communications. This paper motives such development by presenting a use case analysis that illustrates the current and a desirable future state of MSC information communication. The future state, which relies on a standard MSC model can advance the current practice and allow precise and computer-interpretable representation of MSC information.
\end{abstract}

Keywords: manufacturing service, manufacturing service capability, dynamic supply chain.

\section{Introduction}

Accurate information about manufacturing capabilities is essential when forming a manufacturing supply network. Key supplier capability factors are production processes, quality, capacity, cost, and digital information processing ability. These are collectively referred to as manufacturing service capability (MSC) information. Manufacturing service capability (MSC) information must be accurate and easily accessible to the supply chain. Multiple manufacturing communities and enterprises have developed proprietary MSC models. These models differ semantically, structurally, and representationally - they have different taxonomies for categorizing manufacturing services, different representations for expressing production requirements. In addition, the models still rely largely on unstructured data. Therefore, there are challenges in today's industry practices to assemble a manufacturing supply chain. For instance, the current means of matching a customer's requirements to a supplier's capabilities is largely manual and inefficient. Supply chain data are transformed manually throughout the supply chain. In many cases the manufacturing software 
applications are incompatible between the sender and receiver. This can cause data errors and data quality to deteriorate as data are being passed to lower tier suppliers. These situations ultimately impede effective sourcing and can lead to production issues.

The focus of our work is to enhance communication of MSC information carried in the form of MSC description instantiated from a standard MSC model. MSC descriptions may be carried in electronic documents as manufacturing requirements (e.g., RFQ - Request for Quotation) or may be exposed on suppliers' web sites and web-based information-sharing portals as supplier's manufacturing capabilities.

This paper proposes the development of a standard MSC model by presenting a use case analysis that illustrates the current and a desirable, standard-based future state of MSC information communication. The use case scenario provides context for the standard MSC model information requirements. The rest of the paper is structured as follows. In section 2, we provide a RFQ use-case scenario illustrating MSC information that needs to be communicated between a manufacturing customer (customer, for short) and suppliers. In section 3, we analyze the current state where MSC information is communicated using proprietary MSC models and descriptions; and illustrate the desirable future state. Due to space limitation and for simplicity, these proprietary and standard models are illustrated as data elements in tabular form, while the actual standard model is expected to be represented in a formal representation language. We then describe related work in section 4. Finally we provide conclusions and future plans which include developing an MSC model evolution framework and tools as well as working with industry to develop a standard model.

\section{Request for Quote Use Case Scenario}

In this scenario, a customer needs a supplier to produce a custom bearing with specific requirements. Below, two ways in which MSC information (in this case, the manufacturing requirements) can be communicated are shown using product-centric or process-centric MSC descriptions for a custom bearings manufacturing RFQ.

\section{Product-Centric MSC description}

- Bearing Type: Spherical

- Order quantity: 1,000

- Delivery date: Dec 31, 2012

- Application: Military

- Metric: Inch

- Lubrication port: Required

- Sealed: No

- Bore diameter: $15 \mathrm{~cm}$

- Outer diameter: $21.875 \mathrm{~cm}$

- Width: $11.875 \mathrm{~cm}$

- Housing width: $10.3125 \mathrm{~cm}$

- Basic dynamic load rating: $17630 \mathrm{~kg}$

- Basic static load rating: $530612 \mathrm{~kg}$

- Ball material - Alloy steel, heat treated, hard chrome plated.

- Race material - Stainless steel, heat treated

\section{Process-Centric MSC description}

- Order quantity: 1,000

Stock shape: Tubes or Round stock

Material: Alloy steel

Industry: Military

- Required manufacturing processes ○ Turning

- Outside Diameter: $21.875 \mathrm{~cm}$

- Diametric tolerance: $0.125 \mathrm{~mm}$

- Concentricity: $0.025 \mathrm{~mm}$

- Process annealing to $260{ }^{\circ} \mathrm{C}$

- Grinding

- Surface finish: 12 RA

- Outside diameter: $21.875 \mathrm{~cm}$

- Inside diameter: $15 \mathrm{~cm}$

o File format: CATIA version 4 
A product-centric MSC description conveys manufacturing requirements via required product design features and functional properties such as Bearing Type, Lubrication port, and Sealed. On the other hand, the process-centric MSC description conveys manufacturing requirements in terms of process capabilities required to produce a product. Process-centric MSC descriptions may be expressed with processoriented information, resource-oriented information, or combination of the two. The process capabilities include manufacturing process capabilities and information processing capabilities. The process-centric description using process-oriented information describes information in terms of particular manufacturing processes, such as CNC (Computer Numerical Control) machining, EDM (Electric discharge machining); and software functions, such as support for CAD (Computer-Aided Design) file formats or mechanical or design analysis. The process-centric description using resource-oriented information, on the other hand, describes information in terms of available resources including machines, tools, and software (e.g., 3-axis Vertical CNC machining center, specific CAD System).

\section{Current and Future State Analyses of Communication via MSC Descriptions}

This section illustrates the current and desired state of communicating MSC descriptions using the custom bearings example.

\subsection{Product-Centric MSC Models}

The proprietary MSC models, $m 1, m 2$, and $m 3$, shown below illustrate current product-centric MSC models that are used by suppliers to create their MSC descriptions. The custom bearing customer uses these models and descriptions to communicate MSC information - in this case matching manufacturing requirements (from the customer) with capabilities (from suppliers). Below, three proprietary product-centric MSC models for custom bearing and respective properties from the custom bearing manufacturers are analyzed. These three models are respectively based on a proprietary RFQ form on a supplier web site, a proprietary product catalog, and a search form from a supplier search portal.

The $m l$ proprietary MSC model uses a natural language description of the required functions (a lot of data fields have string data types) and proprietary characterization of desired performances and data based on proprietary representation of geometric schematics (of product model).

The $m 2$ proprietary MSC model uses proprietary material and part classifications (i.e., proprietary code lists) and proprietary characterization of desired performances. The model $m 2$ is more limited than the model $m 1$. A number of design features and functionalities in the manufacturing requirements cannot be captured using this $m 2$ model. However, some requirements, such as the Sealed and Application properties, which could not be represented with the model $m l$, are representable with the model $m 2$. These properties are used as part of the customer requirements. 
The last proprietary MSC model, $m 3$, uses proprietary part classification, proprietary characterization of desired performances, and ad hoc classification of material and functional features using proprietary code lists. This model has also a number of issues: it cannot capture all the manufacturing requirements from our example; properties are semantically ambiguous e.g., Load Capacity property does not differentiate between Basic Dynamic Load Rating and Basic Static Load Rating; dimensional properties such as Bore Diameter and Outside Diameter are specified in data value ranges (conveyed via code lists) rather than as precise numeric values; there may be differences in concepts based on the terminology such as Length through Bore vs. Width.

\section{Product-centric MSC model de- rived from RFQ Form $(m 1)$}

- Application: code list

- Quantities to quote: number

- Usage: string

- Grooved for lubrication / Plain: code

- B (Bore diameter): number

- D (Outside diameter): number

- W (Width): number

- H (Housing width): number

- a (Misalignment angle): number

- Material for Ball: string

- Material for Race: string

- Material for Liner: string

- Type of Bearing Loads: string

- Radial Bearing Load: number

- Axial Bearing Load: number

- Operating Temperature: number

- Corrosion: string

- Washdown: string

\section{Product-centric MSC model derived from Product Catalog (m2)}

- Inner Ring Material: code list

- Outer Ring Material: code list

- Maintenance Free: boolean

- Sealed: boolean

- Angle of misalignment: number

- Applications: code list

- Quantity: number
Product-centric MSC model derived from Search Form (m3)

- Design units: code list

- Bore diameter: code list

- Length through Bore: code list

- Outside diameter: code list

- Maximum Angular Misalignment: code list

- Load capacity: code list

- Material: code list

- Self-lubricating: boolean

- Lubrication Port: boolean

- Corrosion Resistant: boolean

- Suitable for Rotating Shaft: boolean

The properties captured in those three presented proprietary product-centric MSC models illustrate the heterogeneities and limitations of how bearing features and functions are communicated. A customer having MSC description of the custom bearing described in section 2 would need to convert his/her MSC description to these various manufacturer MSC information models, if at all possible, to engage in an RFQ transaction and supply chain assembly with potential suppliers. Such communication limitation prevents customers from getting to suppliers with the right capability at the right time with the right effort.

A future state of enhanced MSC information communication for custom bearings can be enabled by a standard product-centric MSC model. Table 1 shows a possible standard MSC model constructed by harmonizing properties from the three analyzed proprietary product-centric MSC models. In this case, free-form text field and proprietary code lists are replaced with standard code lists that may be supplemented by respective ontologies. The proposed model is semantically rich and can cover more bearing characteristics than those necessary to describe the example manufacturing requirements. That is, <no requirement $>$ in Table 1 denotes properties, identified in 
the analyzed proprietary product-centric MSC models, but not required in the example manufacturing requirements. This means the possible standard model is richer in semantics and can support more variations of bearing specifications. Only with a standard MSC model, the customer can effectively communicate with a large pool of suppliers using shared concepts and semantics and be able to get to the right supplier with the right capability at the right time and effort.

Table 1. Possible data elements for standard product-centric MSC model

\begin{tabular}{|c|c|c|}
\hline Property Group & Property: Data Type & $\begin{array}{l}\text { Customer Requirement Map (from the } \\
\text { Product-Centric MSC description) }\end{array}$ \\
\hline \multirow{2}{*}{ Production } & Quantity: number & Order quantity \\
\hline & Expected delivery date: date & Delivery date \\
\hline \multirow{5}{*}{ Dimension } & Design units: standard code list & Metric \\
\hline & Bore diameter: number & Bore diameter \\
\hline & Length through Bore: number & Width \\
\hline & Outside diameter: number & Outer diameter \\
\hline & Housing diameter: number & Housing width \\
\hline \multirow{4}{*}{$\begin{array}{l}\text { Performance } \\
\text { specification }\end{array}$} & Type of Bearing Loads: standard code list & Dynamic load or Static load \\
\hline & Radial Bearing Load: number & Dynamic load or Static load value \\
\hline & Axial Bearing Load: number & $<$ no requirement $>$ \\
\hline & Maximum Angular Misalignment: number & $<$ no requirement $>$ \\
\hline \multirow{3}{*}{ Material } & Ball: standard code list & Ball material \\
\hline & Race: standard code list & Race material \\
\hline & Liner: standard code list & $<$ no requirement $>$ \\
\hline \multirow{4}{*}{ Application } & Type: standard code list & $<$ no requirement $>$ \\
\hline & Application area: standard code list & Application \\
\hline & Usage: string & Usage \\
\hline & Operating temperature: number & $<$ no requirement $>$ \\
\hline \multirow{7}{*}{ Features } & Self-lubricating: boolean & $<$ no requirement $>$ \\
\hline & Lubrication Port: boolean & Lubrication port \\
\hline & Corrosion Resistant: boolean & $<$ no requirement $>$ \\
\hline & Suitable for Rotating Shaft: boolean & $<$ no requirement $>$ \\
\hline & Suitable for Washdown: boolean & $<$ no requirement $>$ \\
\hline & Maintenance-free: boolean & $<$ no requirement $>$ \\
\hline & Sealed: boolean & Sealed \\
\hline
\end{tabular}

\subsection{Process-Centric MSC Models}

Below, three current proprietary process-centric MSC models, $m 4, m 5$, and $m 6$, from three custom bearing manufacturers are shown. The first two are based on proprietary MSC descriptions from web sites of two suppliers. The other is based on a search form of a supplier information sharing portal. The first two use combination of 
process-oriented and resource-oriented information while the other uses only processoriented information.

In $m 4$, a number of manufacturing requirements cannot be specified, including Order quantity, Stock shape, Concentricity tolerance, and Process annealing temperature.

As with $m 4$, the second proprietary MSC model, $m 5$, cannot support a number of manufacturing requirements including Order quantity, Stock shape, Surface grinding finish tolerance, and Process annealing temperature requirement. Furthermore, the annealing process requirement can only be specified using a more generic concept, the heat treatment process.

The last proprietary MSC model, m6, also cannot support a number of manufacturing requirements, including Order quantity, Stock shape, Surface grinding finish tolerance, Process annealing temperature and File format.

Process-centric MSC model derived from Supplier profile $1(\mathrm{~m} 4)$

- Machining Processes: proprietary code $\cdot$ Machining Processes: proprietary list

- Equipment: proprietary code list

- Machinery Axis: number

- Fixturing: proprietary code list

- Maximum Part Diameter (Turning): number with constraint

- Maximum Part Length (Turning): number with constraint

- Maximum Bar Feed Diameter Capacity (Turning): number

- Maximum Swing (Turning): number with constraint

- Maximum Part Size (CNC Milling,

Vertical): formatted string

- Industries: proprietary code list

- Industry Standards: proprietary code list

- Efficiency: proprietary code list

- File Formats: proprietary code list

- Maximum Part Size (CNC Milling,

Horizontal): formatted string

- Production Tolerances (+/-): number with constraint

- CNC Products Type: proprietary code list

- Materials: proprietary code list

- In House Additional Services: proprietary code list

- Additional Services: proprietary code list

- Inspection: proprietary code list

- Quality: proprietary code list

- Inventory Control: proprietary code list

- Production Volume: proprietary code list from Supplier profile 2 (m5) code list

- Equipment: proprietary code list

- Equipment Capabilities: proprietary code list

- Machinery Axis: number

- Fixturing: proprietary code list

- CNC Turned Part Diameter: number

- CNC Turned Part Length: number

- CNC Milled Part Length: number

- CNC Milled Part Width: number

- CNC Milled Part Height: number

- Straightness : number

- Diameter Tolerances (out/in) :

number

- Concentricity : number

- Length Tolerances: number

- Intended applications: proprietary code list

- Materials (Metals) : proprietary code list

- Materials (Plastic Polymers) : pro-

prietary code list

- Secondary Services: proprietary

code list

- Production Volume: proprietary code list

Industries Served: proprietary code list

Industry Standards: proprietary code list

- Lead Times Available: proprietary code list

Process-centric MSC model derived Process-centric MSC

data-set model derived from Search form (m6)

- Capabilities: proprietary code list

- Number of Axes: proprietary code list

- Specialty Machining: proprietary code list

- Diameter Capacity:

proprietary code list

- Length Capacity: proprietary code list

- Micro Machining: boolean

- Materials: proprietary code list

- Secondary Services Offered: proprietary code list

- Additional Services: proprietary code list

- Location: proprietary code list 
A future state of enhanced MSC information communication for custom bearing manufacturing can be enabled by a standard process-centric MSC model. Table 2 shows a possible standard MSC model, which extends a merge of a subset of the properties defined in the three proprietary process-centric MSC models. The standard MSC model would be formal and structured specifications of required manufacturing capabilities and process requirements using shared concepts and semantics. The model is semantically rich and can cover more manufacturing characteristics than those necessary to describe the manufacturing requirements for our custom bearings example. Proprietary code lists are replaced with standard code lists that may be supplemented by respective ontologies. It should be noted $<$ no requirement $>$ signifies the same meaning as that in Table 1.

Table 2. Possible data elements for standard process-centric MSC model

\begin{tabular}{|l|l|}
\hline \multicolumn{1}{|c|}{ Property: Data Type } & $\begin{array}{l}\text { Customer Requirement Map (from the } \\
\text { Process-Centric MSC description) }\end{array}$ \\
\hline Machining process: standard code list & Turning, Grinding \\
\hline Specialty machining process: standard code list & $<$ no requirement $>$ \\
\hline Equipment: standard code list & $<$ no requirement $>$ \\
\hline Machinery axis: number $(2-9)$ & $<$ no requirement $>$ \\
\hline Fixturing: standard code list & $<$ no requirement $>$ \\
\hline Turning max part diameter: number & 21.875 cm \\
\hline Turning max part length: number & $<$ no requirement $>$ \\
\hline Turning max bar feed diameter: number & $<$ no requirement $>$ \\
\hline Turning Swing: number & $<$ no requirement $>$ \\
\hline Milling max part length: number & $<$ no requirement $>$ \\
\hline Milling max part width: number & $<$ no requirement $>$ \\
\hline Milling part height: number & $<$ no requirement $>$ \\
\hline Micro machining: number & $<$ no requirement $>$ \\
\hline Straightness: number & $<$ no requirement $>$ \\
\hline Diameter tolerances: number & 0.125 mm \\
\hline Concentricity: number & 0.025 mm \\
\hline Length tolerances: number & $<$ no requirement $>$ \\
\hline Materials: standard code list & Alloy Steel, Stainless Steel \\
\hline Secondary services: complex structure & Heat treating to $260{ }^{\circ} \mathrm{C}$ \\
\hline Quality control capabilities: standard code list & $<$ no requirement $>$ \\
\hline Inventory control capabilities: standard code list & $<$ no requirement $>$ \\
\hline Lead time capabilities: standard code list & $<$ no requirement $>$ \\
\hline Inspection capabilities: standard code list & $<$ no requirement $>$ \\
\hline Production volume: standard code list & Medium \\
\hline Industries served: standard code list & Military \\
\hline Product focus: standard code list & Bearing \\
\hline Industry standards: standard code list & $<$ no requirement $>$ \\
\hline File formats accepted: standard code list & Catia version 4 \\
\hline Location: standard code list & $<$ no requirement $>$ \\
\hline & \\
\hline
\end{tabular}




\section{$4 \quad$ Related Work}

There are efforts towards standardization of MSC model and its semanticenhancement. Ameri and Dutta [1] have built Manufacturing Service OWL (Web Ontology Language) ontology to provide for shared semantics of the process-centric MSC information. Jang et al. [7] developed similar OWL-based manufacturing service ontology, however, in product-centric way using machining features. A resource model developed by Vichare et al. [8] can be a basis for shared semantics of the resource-oriented process-centric MSC information. Ontology for representing fixture design knowledge in Ameri and Summers [2] can also provide a basis for resourceoriented MSC information of fixtures. In addition to these ontology-based efforts, other related standardization efforts include ISO 14649 (STEP-NC) [5] standard for machining features, ISO 15331 [6] standard for representing machining resources, ISO 13399 [4] standard for representing cutting tool information, and ASME B5.59-2 [3] standard for describing the performance and capabilities of milling and turning machines.

\section{Conclusion and Future Work}

This paper highlights the inefficiency in communication of MSC information via MSC descriptions between suppliers and manufacturers. Communicating MSC information is important for enabling supply chain agility. The lack of effective standards is a barrier to improving MSC information communication. As the first stage in developing a standard, this paper provided an illustrative use case analysis of the current and future communications of MSC information via respective MSC models. MSC descriptions are characterized into product- and process-centric MSC descriptions, and the process-centric MSC descriptions may be specified with resource- and/or process-oriented information. The MSC descriptions conforming to an advanced and standardized MSC model should significantly enhance current practice in communicating requirements and capabilities for manufacturing services. Our immediate future work will include more comprehensive MSC information requirements analysis, followed by creating and validating the target standard MSC model that enables semantically precise representation of product- and process-centric MSC descriptions.

\section{Disclaimer}

Certain commercial software products are identified in this paper. These products were used only for demonstration purposes. This use does not imply approval or endorsement by NIST, nor does it imply these products are necessarily the best available for the purpose. 


\section{References}

1. Ameri, F., Dutta, D.: An Upper Ontology for Manufacturing Service Description. In: ASME Conference Proceeding 2006, p. 651 (2006), doi:10.1115/DETC2006-99600

2. Ameri, F., Summers, J.D.: An ontology for representation of fixture design knowledge. Computer Aided Design and Applications 5, 601-611 (2008)

3. ASME B5.59-2. Information technology for machine tools - part 2: Data specification for properties of machine tools for milling and turning, draft (2005)

4. ISO 13399-1 Cutting tool data representation and exchange - part 1: Overview, fundamental principles and general information model (2006)

5. ISO 14649-1 Industrial automation systems and integration - physical device con-trol - data model for computerized numerical controllers, part 1: Overview and fundamental principles (2002)

6. ISO 15531-1 Industrial automation systems and integration - industrial manufac-turing management data - part 1: General overview (2003)

7. Jang, J., Jeong, B., Kulvatunyou, B.S., Chang, J., Cho, H.: Discovering and Integrating Distributed Manufacturing Services with Semantic Manufacturing Capability Profiles. International Journal of Computer-Integrated Manufacturing 21(6), 631-646 (2008)

8. Vichare, P., Nassehi, A., Kumar, S., Newman, S.: A Unified Manufacturing Resource Model for Representation of CNC Machining Systems. In: 18th International Conference on Flexible Automation and Intelligent Manufacturing (FAIM), Skövde, June 06-July 02 (2008) 